\title{
Desafíos educativos en ambientes virtuales: escuelas rurales y urbanas
}

\section{Educational challenges in virtual environments: rural and urban schools}

\author{
Perla Meléndez Grijalva* \\ Universidad Pedagógica Nacional del Estado de Chihuahua, Chihuahua, México \\ ORCID: https://orcid.org/0000-0003-1239-0774 \\ Celia Carrera Hernández \\ Universidad Pedagógica Nacional del Estado de Chihuahua, Chihuahua, México \\ ORCID: https://orcid.org/0000-0002-2444-2204 \\ Josefina Madrigal Luna \\ Universidad Pedagógica Nacional del Estado de Chihuahua, Chihuahua, México \\ ORCID: https://orcid.org/0000-0003-2190-3164
}

*Correspondencia:

Email: pmelendez@upnech.edu.mx
Citar como:

Meléndez, P., Carrera, C., \& Madrigal, J. (2021). Desafíos educativos en ambientes virtuales: escuelas rurales y urbanas. Propósitos y Representaciones, 9(3), e1333. http://dx.doi.org/10.20511/pyr2021.v9n3.1333

(c) Universidad San Ignacio de Loyola, Facultad de Humanidades, Carrera de Psicología, 2021. 


\section{Resumen}

Se presentan los resultados de una investigación que indagó en las percepciones y experiencias de estudiantes universitarios en contextos urbanos y rurales, a fin de conocer cómo viven su educación en una modalidad virtual bajo un contexto de pandemia, considerando tres categorias: condiciones pedagógicas, materiales y emocionales. Fue un estudio cualitativo a partir del método fenomenológico, se aplicaron entrevistas a profundidad y análisis documental. Participaron doce universitarios de zonas rurales y quince de urbanas en el Estado de Chihuahua. Los resultados indican que en ambos contextos se percibió una educación deficiente, con prácticas docentes tradicionales y aburridas, con aprendizajes por debajo de lo esperado y retroalimentación y comunicación poco efectiva; se presentó baja motivación y sentimientos recurrentes de miedo, tristeza y desesperanza; la mayor preocupación en contextos urbanos fue el riesgo de contagio y el aislamiento social, en rurales los problemas de conectividad y la pobreza económica. Sobre los recursos materiales, los estudiantes en contextos rurales tuvieron mayores carencias, menos de la mitad contaban con computadora e internet. Por otro lado, se encontró un desarrollo de habilidades tecnológicas y de autogestión del aprendizaje como la administración del tiempo y los recursos, la organización de actividades y la autoconstrucción del conocimiento. Se concluye que la educación virtual se desarrolla bajo un paradigma tradicional, lo que hace necesario fortalecer no sólo el acceso a tecnologías, también promover la motivacion, participación e interacciones entre estudiantes y transformar las prácticas pasivas por experiencias organizadas, flexibles y con impacto social, actitudinal y cognitivo.

Palabras clave: Educación virtual; Percepciones; Estudiantes; Prácticas docentes.

\section{Summary}

The results of an investigation that investigated the perceptions and experiences of university students in urban and rural contexts are presented, in order to know how they live their education in a virtual mode under a pandemic context, considering three categories: pedagogical, material and emotional It was a qualitative study based on the phenomenological method, in-depth interviews and documentary analysis were applied. Twelve university students from rural areas and 15 from urban areas in the State of Chihuahua participated. The results indicate that in both contexts a deficient education was perceived, with traditional and boring teaching practices, with lower than expected learning and ineffective feedback and communication; low motivation and recurrent feelings of fear, sadness and hopelessness were presented. The greatest concern in urban contexts was the risk of contagion and social isolation, in rural the problems of connectivity and economic poverty. Regarding material resources, students in rural contexts had greater deficiencies, less than half had a computer and internet. On the other hand, it was found a development of technological skills and self-management of learning such as time and resource management, the organization of activities and the self-construction of knowledge. It is concluded that virtual education is developed under a traditional paradigm, which makes it necessary to strengthen not only access to technologies, but also to promote motivation, participation and interactions among students and transform passive practices through organized, flexible experiences with social impact, attitudinal and cognitive.

Keywords: Virtual education; Perceptions; Students; Teaching practices.

\section{Introducción}

Ante la situación inédita provocada por la pandemia del COVID 19, que ha llevado a las autoridades educativas de todos los países a la sustitución de la modalidad presencial de las clases en los distintos niveles por una modalidad a distancia y/o virtual, ha hecho visible la precariedad en que muchas escuelas, sobre todo de zonas rurales y urbanas en contextos marginados, se 
encuentran, acrecentando las desigualdades en los procesos de enseñanza-aprendizaje de profesores y estudiantes.

El proceso educativo ha tenido que ajustarse y adaptarse a esta modalidad virtual y/o a distancia, desconocida por muchos, y que requiere del acceso a recursos tecnológicos así como habilidades y conocimientos para implementarlos en el ámbito académico, adecuaciones a la metodología de la enseñanza, desarrollo de competencias emocionales para afrontar el cambio y la pandemia misma, la organización de tiempos, espacios y actividades para trasladar la escuela al hogar, entre otros, convirtiendo a la educación en un desafio para autoridades, profesores, alumnos y familia.

Se hace necesario conocer, desde la voz de los actores involucrados, las experiencias y percepciones que esta situación les ha traído, pues la educación es un fenómeno complejo que va más allá de aspectos tangibles como los conocimientos y habilidades plasmados en las calificaciones de los estudiantes; conocer cómo viven y sienten el proceso educativo que actualmente se hace presente en todas las escuelas, para fines de este estudio escuelas de nivel superior, permite entender qué, cómo y porqué ocurren fenómenos que aparentemente son iguales para todos, pero que impactan y se desarrollan dependiendo el contexto y sus características.

Esta investigación pretende describir, interpretar y contrastar la percepción de estudiantes universitarios de escuelas urbanas y escuelas rurales, respecto a tres categorías $\mathrm{o}$ dimensiones,mismas que emergieron de un grupo focal y fueron a priori para el diseño de una entrevista a profundidad: las condiciones emocionales para afrontar una metodología virtual de educación en un contexto global de pandemia; las condiciones materiales y de recursos para atender esta modalidad desde sus hogares; y las condiciones pedagógicas para lograr los aprendizajes esperados en el plan de estudios que cursan bajo la modalidad virtual y/o a distancia.

\section{Escenarios educativos ante la pandemia COVID 19}

A principios del 2020 oficialmente se declara una situación de pandemia por el virus COVID-19 en todo el mundo, trayendo consigo una serie de estrategias gubernamentales para evitar la propagación del mismo. El sistema educativo no permaneció ajeno. El confinamiento causó un cambio improvisado de una modalidad educativa presencial a ambientes de aprendizaje virtual, generando crisis a autoridades, docentes, estudiantes, familia y comunidad en general.

Los nuevos tiempos demandarían una transformación en los procesos de enseñanza y aprendizaje que muchas instituciones educativas no estaban preparadas para afrontar. Adecuar estos cambios se convirtió en un reto que involucra no solo a los docentes y alumnos, sino a toda la sociedad. Trasladar las clases de un aula al hogar, implicaría no sólo contar con los recursos tecnológicos y financieros, sino con el tiempo y trabajo de los padres o tutores para apoyar en las tareas a sus hijos, sobretodo los más pequeños, así como el cuidado de ellos de tiempo completo.

Por otro lado, adecuar los contenidos y la metodología de trabajo, también se convirtió en un desafío para los docentes, algunos de ellos sin conocimiento de la didáctica para la enseñanza virtual e incluso, sin las habilidades en el manejo de los recursos digitales. Además, según un informe que presentan La Comisión Económica para América Latina y el Caribe (CEPAL) y La Organización de las Naciones Unidas para la Educación, la Ciencia y la Cultura (UNESCO):

La necesidad de ajuste a las condiciones de la educación a distancia se ha traducido, asimismo, en un conjunto de responsabilidades y exigencias que aumentan significativamente el tiempo de trabajo que las y los docentes requieren para preparar las clases, asegurar conexiones adecuadas y hacer seguimiento a sus estudiantes en formatos diversos (2020, p. 10). 
Son pocos los países de la región centro y sur de América que cuentan con estrategias nacionales de educación por medios digitales con un modelo que aproveche las tecnologías de información y comunicación (TIC), aún y cuando en la Agenda 2030 para el Desarrollo Sostenible se incorpora el Marco de competencias de los docentes en materia de TIC, que incluye 18 competencias de la práctica, siguen siendo limitadas las instituciones que han transformado sus procesos de formación docente en lo referente a estas competencias, necesarias para la educación del siglo XXI (UNESCO, 2019, citado por CEPAL-UNESCO, 2020).

En un estudio realizado por la Organización Valora (2020), encuentra que en México, aún y cuando autoridades educativas, profesores y padres de familia hacen lo mejor que pueden con lo que tienen, hay una dificultad para lograr aprendizajes significativos a distancia por falta de iniciativas pertinentes y por la escasa formación y recursos de profesores, estudiantes y familias; hay desigualdades en el acceso a bienes culturales entre estudiantes de escuelas públicas y privadas y de regiones rurales y urbanas; el incremento del estrés ante la incertidumbre que se vive; la disminución de oportunidades de aprendizaje para niños en contextos vulnerables, entre otros aspectos que afectan la calidad educativa sobre todo para los más desprotegidos (citado por Ruiz, 2020).

La pandemia ha hecho visible cosas que se sabían pero no se atendían, como la falta de formación de profesores en el uso de las TIC y en la didáctica en ambientes virtuales, pues ante una menor presencialidad se hace necesaria una mayor fundamentación didáctica que permita diversificar las alternativas de educación, sobre todo para los más vulnerables; en el aspecto emocional, se necesita promover la formación cívica y ética en casa para mejorar la convivencia y el desarrollo socioemocional; el hogar se convierte en la nueva escuela, y los padres requieren apoyo para que enseñen de acuerdo a sus posibilidades; estos nuevos desafios, que se caracterizan por altas incertidumbres y transformaciones constantes en el entorno, hacen urgente estimular en profesores y estudiantes la capacidad de gestión de los cambios (Pérez, Suárez y Rosillo, 2018).

Educar en un escenario con las condiciones arriba mencionadas, llama, no sólo al sector educativo, sino a la sociedad en general, a actuar de manera organizada y en conjunto. Reimers y Schleicher (2020), proponen algunas alternativas que guíen a autoridades locales y nacionales, como establecer un grupo de trabajo integrado por representantes de diferentes componentes del sistema educativo; definir los principios que guiarán la estrategia, como proteger la salud, garantizar aprendizaje y proporcionar apoyo emocional; definir claramente roles y expectativas de los docentes y estudiantes; definir mecanismos de comunicación efectiva; asegurar el apoyo a estudiantes y familias más vulnerables, entre otros.

\section{La educación virtual: un nuevo paradigma de enseñanza y aprendizaje}

Ante la diversidad de realidades en que se encuentran estudiantes y profesores, es necesario asumir un cambio en la forma de entender el aprendizaje y la enseñanza. Al respecto, Arango, Díaz y Zuleta (2018) señalan que la educación superior en ambientes virtuales debe ser un agente movilizador de innovación y transformación del contexto social, con una enseñanza que favorezca la autonomía del estudiante y deje de lado el viejo paradigma centrado en el aula y la actividad del profesor.

La educación virtual debe garantizar la inclusión de las Tecnologías de la Información y la Comunicación (TIC) en los procesos académicos de manera intencionada, y debe transformar los métodos pedagógicos para permitir que las personas diseñen su ambiente personal de aprendizaje, es decir, que este último responda hacia las diferencias y las necesidades individuales, como también hacia los intereses colectivos (Díaz, Andrade, Hincapié y Uribe, 2021, s/p). 
A diferencia de la modalidad presencial, caracterizada por una visión centrada en el currículo con prácticas escolares tradicionales, la enseñanza virtual interactiva se caracteriza por ofrecer versatilidad de herramientas y metodologías que sean flexibles para personalizarse y adaptarse a las necesidades particulares de cada estudiante o grupo de estudiantes, lo que les va a permitir aprender a autogestionar el conocimiento, va propiciar el aprendizaje cooperativo y favorecerá la interacción con la comunidad inmediata y con otras más lejanas, rescatando experiencias del mundo entero (Zamora y Hernández, 2015).

Los espacios virtuales superan los espacios físicos y temporales, unen contextos formales e informales de aprendizaje, potencian un aprendizaje constructivista, activo, variado en métodos y representaciones, integra tareas cooperativas, busca la autonomía del alumno para que éste navegue en un entorno conectado por el mundo, pero organizado de acuerdo al contenido curricular. Esta innovación involucra nuevas formas de comportamiento y de acercamiento a los estudiantes (Pérez, Suárez y Rosillo, 2018).

Este nuevo paradigma intenta formar personas que observen con sentido crítico su realidad, que esten abiertos al cambio y que desafíen los obstáculos para convertir las amenazas en oportunidades, cuidando fortalecer la interacción y el intercambio de experiencias para la toma de decisiones asertivas que permitan enfrentar la complejidad de los nuevos tiempos (Guzmán, Vázquez y Escamilla, 2020).

Sumado a lo anterior, surgen pedagogías emergentes en ambientes virtuales de aprendizaje, con metodologías activas, que requieren prácticas innovadoras que hagan simbiosis entre la tecnología y la pedagogía, pues la primera permite el acceso a datos inimaginables en todas las disciplinas y áreas del conocimiento, pero es la segunda la que promueve que el estudiante no sea un receptor pasivo de información, sino que aprenda a administrar, adquirir, analizar, inferir, planificar, a partir de esos datos (Aparicio y Ostos, 2021).

Los espacios virtuales, entonces, permiten acceder a redes inimaginables de información y conocimientos, mismos que pueden compartirse entre los que interactúan, en este caso docente y alumnos, pero el docente es quien tiene que asegurarse que se dé el proceso de difusión y que se transforme en conocimientos, que se comparta y enriquezca, se le otorgue un sentido y aplicabilidad y, finalmente, puedan profundizar en la comprensión de los temas abordados.

\section{Objetivo del estudio}

Comprender cómo viven los estudiantes de pregrado, de universidades ubicadas en contextos rurales y urbanos, su proceso de aprendizaje en una modalidad educativa virtual y/o a distancia, bajo un contexto global de confinamiento por la pandemia COVID 19.

\section{Preguntas de investigación}

¿Cuáles emociones perciben con mayor frecuencia los estudiantes de licenciatura de universidades ubicadas en contextos rurales y urbanos, al momento de afrontar una metodología virtual de educación en un contexto global de pandemia?

¿Cuáles son las condiciones materiales y de recursos de los estudiantes que viven en contextos rurales y los que están en contextos urbanos, para atender esta modalidad de educación virtual, desde sus hogares?

¿Cómo perciben y valoran, los estudiantes de contextos rurales y contextos urbanos, las condiciones pedagógicas vividas bajo la modalidad virtual y/o a distancia, para lograr los aprendizajes esperados en el plan de estudios que cursan? 


\section{Método}

El estudio se desarrolla bajo un paradigma hermenéutico interpretativo, con un enfoque cualitativo, mediante el método fenomenológico, pues su finalidad es comprender cómo perciben y experimentan los estudiantes de escuelas urbanas y rurales la educación virtual y/o a distancia en tiempos de pandemia, profundizando en el significado e interpretación que le dan, para luego contrastar estas percepciones, según el contexto, e identificar sus coincidencias y/o diferencias. La fenomenología, en palabras de Heidegger (2006), es el estudio que permite comprender lo que significa para un sujeto vivir una situación o fenómeno determinado.

\section{Participantes}

Para la selección de los participantes, se consideraron aquellos que estuvieran inscritos a un programa de licenciatura en la Universidad Pedagógica Nacional del Estado de Chihuahua, por ser una institución con Campus Universitarios presentes en 11 municipios del Estado, algunos de ellos ubicados en zonas rurales y serranas y otros en zonas urbanas. La invitación fue abierta para todo el alumnado y se hizo por medio de los Directivos o autoridades de cada Campus, por escrito, mediante una carta de invitación, explicando el propósito del estudio. Los interesados se comunicaron vía correo electrónico o celular con los responsables de esta investigación. En total se tuvo comunicación con 28 estudiantes de los Campus ubicados en la zona serrana del Estado de Chihuahua y considerada población rural; y 31 estudiantes que asisten a los Campus ubicados en zonas urbanas como las ciudades de Chihuahua y Parral. Por las condiciones y la disponibilidad de los informantes desertaron algunos, quedando al final, para participar en el estudio, 12 estudiantes de zonas rurales y 15 de urbanas.

El 55.55\% de los sujetos participantes estudian en un contexto urbano (campus de Chihuahua y Parral) y un 44.44\% rural (Campus Creel y Guachochi). El 63\% del total se encontraban cursando la licenciatura en intervención educativa y el 37\% la de pedagogía. El 59\% fueron mujeres y el $41 \%$ hombres. $33 \%$ de los estudiantes cursaban el tercer semestre, $26 \%$ el quinto semestre y $41 \%$ el séptimo. El nivel socioeconómico de los estudiantes es predominantemente bajo, siendo sólo $18 \%$ de ellos de nivel medio.

\section{Instrumentos}

Se utiliza la técnica de la entrevista, individual y grupal, con el apoyo de recursos tecnológicos como la aplicación de Zoom y Meet dado que por la pandemia no es posible la modalidad presencial. También se hace un análisis documental que permite revisar material bibliográfico diverso relacionado con el tema y propósito del estudio.

Los instrumentos son una guía de preguntas semiestructuradas para las entrevistas y, fichas textuales para el análisis documental. La construcción de la entrevista individual surge de los resultados de un grupo focal o entrevista grupal con 12 estudiantes de pregrado quienes, durante sus clases virtuales en el curso de seminario de investigación, impartida por uno de los responsables de este estudio, comparten sus experiencias educativas en la modalidad virtual y/o a distancia en relación a tres dimensiones: fortalezas, debilidades y sugerencias para la mejora del proceso de enseñanza-aprendizaje y desarrollo de competencias profesionales.

El análisis de estas experiencias compartidas permitió identificar regularidades recurrentes que fueron organizadas en categorías apriorísticas y sus subcategorías para formular las preguntas que guían la entrevista individual aplicada en un segundo momento. Esta entrevista individual, antes de ser aplicada, se sometió a un proceso de validez a través del método de consulta a expertos y, considerando sus comentarios, fue reelaborada y aplicada a los participantes. 
La entrevista consta de una primera parte donde se recogen algunos datos sociodemográficos. Luego se abordan las tres categorías con sus subcategorías, donde se elaboran preguntas para cada una, que permitan al informante ampliar o profundizar en ellas (Ver Tabla $1)$.

\section{Tabla 1.}

Preguntas de investigación, categorías, subcategorías y preguntas guía de entrevista

\begin{tabular}{llll}
\hline $\begin{array}{l}\text { Preguntas de } \\
\text { investigación }\end{array}$ & Categorías & Subcategorías & Preguntas guía de entrevista \\
\hline
\end{tabular}

¿Cómo perciben y

valoran, los

estudiantes de contextos rurales y

contextos urbanos, las condiciones pedagógicas vividas bajo la modalidad virtual y/o a distancia, para lograr los aprendizajes esperados en el plan de estudios que cursan?
Estrategias y técnicas pedagógicas del docente

Comunicación alumno-docente

Condiciones pedagógicas

Manejo de las TIC del profesor y del alumno

Retroalimentación por parte del docente

Habilidades del alumno para el autoaprendizaje
¿Cuáles fueron las estrategias didácticas más comunes que utilizaron tus maestros?, ¿Consideras que fueron novedosas e interesantes?

¿Favorecieron tus aprendizajes? ¿Porqué?

¿Cuáles fueron los medios y las estrategias que tuvieron durantes sus clases para mantener comunicación con profesores y compañeros? ¿Qué tan eficiente consideras que fue la comunicación? ¿Porqué? ¿Cuáles fueron los obstáculos que no favorecieron la comunicación?

¿Cuáles recursos tecnológicos sabías utilizar para tus estudios, antes de la pandemia? ¿Cuáles has aprendido? ¿Consideras que utilizar las TIC favorece tu aprendizaje? ¿Porqué? ¿Qué opinas sobre las habilidades tecnológicas de tus profesores?

Cuando te quedas con dudas sobre un tema, ¿cómo las resuelves? ¿Qué estrategias utilizan en esta modalidad para retroalimentar y/o revisar las actividades? ¿Cómo valoras el proceso de retroalimentación? ¿Porqué?

¿Cuáles han sido los aprendizajes, además de los académicos, que te ha dejado esta experiencia? ¿Cuáles son los desafios más importantes que has tenido para aprender en esta modalidad? ¿Qué fortalezas y dificultades has experimentado en tu proceso de aprendizaje bajo esta modalidad? ¿Has sido capaz de aprender o resolver dudas por ti mismo? Puedes dar unos ejemplos

¿Te sientes motivado para estudiar y tomar clases en modalidad virtual? ¿Porqué? 
estudiantes de

licenciatura de

universidades

ubicadas en contextos

rurales y urbanos, al

momento de afrontar

una metodología

virtual de educación

en un contexto global

de pandemia?

Situación emocional

¿Cómo te has sentido emocional y anímicamente en tus clases virtuales? ¿Cuál es tu principal preocupación ante este fenómeno que se vive? ¿Qué sentimientos recurrentes has experimentado al estar en confinamiento? ¿Cómo han manejado situaciones como el estrés, ansiedad, etc.? ¿Cuál es el ambiente que se vive en casa?

¿Cuáles son las condiciones materiales y de recursos de los estudiantes que viven en contextos rurales y los que están en contextos urbanos, para atender esta modalidad de educación virtual, desde sus hogares?
Condiciones materiales y de recursos
Equipo de cómputo

Internet

Área de estudio
¿Cuentas con computadora o laptop para hacer tus actividades académicas? Especifica

¿Tienes conectividad en tu casa para las clases o tareas virtuales? En caso negativo, ¿cómo le haces para entregar trabajos o videollamadas?

¿Dónde realizas tus tareas o videollamadas? ¿Consideras que es un lugar adecuado para estudiar? ¿Porqué?

Fuente: Elaboración propia.

\section{Procedimiento}

Para la aplicación de la entrevista, se les informó a los universitarios su confidencialidad, propósito y fines meramente académicos del estudio. Se calendarizó, ajustando de dos a tres entrevistas diarias en horarios diversos, según las condiciones de los estudiantes y de los tres investigadores de este proyecto, durante los meses de octubre y noviembre del 2020. En algunos casos se tuvo que programar una segunda entrevista dado que la información recabada no profundizaba lo suficiente para atender los objetivos del estudio. Se utilizó el Zoom y el Meet y, en algunos casos de estudiantes de zonas rurales, se utilizó el telefono por la falta de acceso a internet en esas comunidades; tanto las entrevistas como las llamadas se grabaron, con el consentimiento de los informantes y, a partir de estas grabaciones, se procedió a la transcripción para su posterior análisis.

\section{Resultados}

Con apoyo del programa Atlas. Ti se transcribieron las expresiones de todos los sujetos entrevistados y se organizaron a partir de las tres categorías identificadas y sus subcategorias. 


\section{Condiciones pedagógicas en que se desarrolló el proceso de enseñanza-aprendizaje.}

Esta categoría recoge las experiencias escolares que los estudiantes tuvieron durante el semestre agosto-diciembre 2020, en una modalidad virtual y/o a distancia y lo que piensan sobre esta modalidad en relación con su desempeño académico y con las prácticas pedagógicas de sus profesores. Se presenta un análisis de las subcategorías:

\section{Estrategias y técnicas pedagógicas del docente.}

En esta subcategoría se analizan las expresiones de los participantes en relación con las estrategias y la metodología con que sus maestros impartieron las clases y qué tanto aportaron a su formación. $\mathrm{Al}$ respecto, los estudiantes, tanto de zonas rurales como urbanas, refieren que las clases fueron aburridas y en muchas ocasiones las actividades no eran suficientes o pertinentes para comprender los contenidos a profundidad.

Algunos de los docentes no se encuentran actualizados e imparten sus clases de manera aburrida y complicada, por lo que uno se termina volviendo autodidáctico. (Sujeto 12, contexto urbano-CU).

Es dificil entender los temas, casi no hay explicación solo te piden leer y escribir algo, yo digo que siempre se necesita que expliquen y es más claro en persona (Sujeto 24, CR).

Las actividades siempre son iguales, de hecho, se parecen a las que hacíamos en presencial pero ahora sin poder socializar con los compañeros por lo que te quedas con tus mismas ideas y no aprendes de otros, además son actividades donde duras muchas horas sentado frente a la computadora leyendo cosas que no entiendes y que terminas haciendo solo por cumplir sin de verdad aprender algo (Sujeto 3, CU).

Muchos profes no eran didácticos, encargaban las mismas actividades con las que no alcanzabas a ver bien un tema y comprenderlo, daban explicaciones confusas o incompletas, eran tareas muy largas y saturadas, las hacías solo para no reprobar, pero creo que el aprendizaje fue muy poco (Sujeto 21, CR).

Se observa en las expresiones de los estudiantes insatisfacción debido a una percepción de las prácticas de sus docentes muy apegadas a las prácticas tradicionales que han llevado en sus clases presenciales, se reproducen formas ortodoxas, expositivas y a veces carentes de flexibilidad, con una participación pasiva y receptora por parte del alumnado lo que conlleva al desinterés y falta de compromiso en su proceso de aprendizaje. Hay carencia de elementos didácticos que favorezcan la comprensión de los contenidos además de actividades con una presentación poco atractiva y con insuficiente integración multimedia.

Desde otra perspectiva, hubo estudiantes, menos que lo anteriores, que comentan buenas experiencias pedagógicas en estos contextos virtuales, haciendo alusión a que esta modalidad les ha permitido aprender y/o mejorar sus conocimientos y habilidades tecnológicas y ha estimulado el deseo por aprender más allá de lo que el profesor les indica, desarrollando la autodisciplina y el autoaprendizaje. También expresaron que este fenómeno ha sido una experiencia que les ayuda a reflexionar y mirar desde una perspectiva más crítica lo que ocurre a su alrededor, adaptarse a los cambios con una actitud positiva y buscar soluciones o alternativas para sobrellevar los problemas académicos, sociales y personales que se les han presentado.

Me sentí más comprometida con lo que estoy haciendo, el tomarle valor y sentido a lo que estoy estudiando, realmente comprometerme y a pesar de las muchas dificultades y obstáculos que se me presentaron, siempre busque la manera de resolverlos y salir adelante, cumplir en forma y tiempo con cada uno de los trabajos asignados (Sujeto 6, CU). 
Aunque al principio me resultó difícil y estresante porque se me juntaba todo el trabajo al final y no alcanzaba a terminar, esto también me sirvió para aprender a organizarme y administrar mi tiempo, siento que aprendí a planear no solo mis cosas de la escuela también otras actividades que nunca hacia o terminaba por desorganizada (Sujeto 10, CU).

La verdad no me gusta la modalidad virtual porque donde vivo se batalla mucho con el Internet, pero tengo que reconocer que me he hecho más independiente, ahora si no entiendo algo lo busco en el google y hasta comparo lo que dice un documento con otro y creo que eso también me ha hecho más inteligente y más analítico (Sujeto 26, CR).

Dentro de lo positivo, los estudiantes reconocen un desarrollo de habilidades tecnológicas y de autogestión del aprendizaje. Al respecto, se destaca un aumento en la administración del tiempo y los recursos, la organización de las actividades y la autoconstrucción del conocimiento.

\section{Comunicación alumno-docente.}

En esta subcategoría se describe la manera en que se estableció y fluyó la comunicación profesorestudiante y estudiante-estudiante, analizando la valoración que hacen los alumnos de ésta. En términos generales, hubo coincidencia en los comentarios de los participantes, sin importar su contexto urbano o rural: fue más unilateral que de diálogo y había poca claridad en las ideas sobre todo cuando fue por vía escrita. La dinámica más común fue el docente mandar un mensaje instruccional, el estudiante atiende y el maestro revisa y avisa si fue correcto o no. Los recursos más utilizados fueron los mensajes de texto, whatsapp y correos electrónicos.

Sin embargo, por las mejores condiciones de internet en los contextos urbanos, tuvieron mayor comunicación con los docentes, pues hubo video conferencias a través de zoom o meet, al menos una por semana con algunos de ellos. También utilizaron la plataforma Moodle, que facilitaba el envío de las actividades, su evaluación y retroalimentación. Sin embargo, en algunos casos, a pesar de las distintas herramientas a las que tenían acceso, no se daba una comunicación horizontal, el maestro más bien explicaba, decía que estaba bien o mal, pero no había tiempo para un diálogo que asegurara que el estudiante había aclarado las dudas o errores.

Sobre la comunicación, creo que fue un poco menos que cuando ibamos a la escuela ya que de esta manera tan solo era recibir instrucciones, hacer los trabajos y regresarlos al profe para que los calificara, a veces no nos decían porqué estuvo mal o qué faltó y cuando sí nos decían, ya no habia oportunidad de corregir o de que nos explicaran otra vez (Sujeto 25, CR)

Casi todos los maestros tuvieron la disponibilidad para contestar mensajes o llamadas cuando teniamos dudas de cómo hacer una actividad, hasta en horario que no era de la clase, pero cuando teniamos las videollamadas no alcanzaba el tiempo para opinar o preguntar (Sujeto 2, $C U)$.

Hubo una maestra que ponia foros para que opinaramos lo que otros compañeros habian puesto de algun tema, creo que fue de las actividades donde más pudimos socializar o conocer lo que los demás pensaban (Sujeto 9, CU).

Se mantuvo contacto con los maestros, a veces llamadas o mensajes, con los compañeros también se tenía un grupo de whatsapp y ahí nos preguntabamos de las tareas, faltó más interacción sobre los temas, la comunicación era más bien para indicar cómo hacer el trabajo (Sujeto 22, CR).

Por la forma en que nos comunicabamos no se llegaba realmente a una reflexión, por decirlo así, de la importancia o la conexión que tenían las temáticas con el curso (Sujeto 18, CR). 
Se percibe en los comentarios de los estudiantes, que las interacciones fueron escasas y de tipo instruccional más que dialógico y, las habilidades comunicativas, no se favorecieron pues los espacios para socializar entre compañeros estuvieron limitados por el tiempo y los mismos recursos tecnológicos, a excepción de los foros que algunos profesores utilizaron. En este sentido, hay expresiones de los entrevistados que dejan ver su preocupación al no lograr una comunicación efectiva con profesores y compañeros, generando un sentimiento de fracaso en la construcción de sus conocimientos.

Finalmente, se puede observar que, en el caso de los contextos urbanos, las relaciones interpersonales fueron más favorables porque tuvieron la oportunidad de establecer comunicación sincrónica a través de videollamadas.

\section{Manejo de las TIC del alumno.}

Se refiere a las habilidades tecnológicas que los estudiantes consideran que tienen o no, tanto ellos como sus profesores, para trabajar en una modalidad virtual. Entre los comentarios más recurrentes, de ambos contextos, hacen mención que les significó un desafio, pues a pesar de saber utilizar una computadora, navegadores de internet, redes sociales, correos electronicos, entre otras herramientas, aprendieron dos cosas en específico que los entrevistados valoran: hacer búsquedas en diferentes bases de datos de información científica y académica y, a utilizar plataformas como el Moodle, la cual consideran que fue atractiva, novedosa y funcional. También coinciden en que sus profesores, la mayoría, aprendió rápido a utilizar los recursos digitales.

Algunos de los comentarios fueron:

Pensaba que sabía usar las TIC, pero me dí cuenta que el internet solo lo usaba para andar en redes sociales, ahora le encuentro otro sentido más productivo y sobretodo, conozco otras plataformas más confiables como google académico para buscar información verídica para mis tareas (Sujeto 25, CR).

Me gusta mucho todo lo que tiene que ver con la tecnología y creo que soy bueno para la computadora y conozco muchas App de todo tipo y las que no conozco luego luego les aprendo (Sujeto 2, CU).

Yo creo que nosotros como estudiantes nos adaptamos más fácil al uso de las TIC para nuestras clases virtuales, muchos ya sabíamos y lo que no conociamos como las plataformas moodle, fue rápido aprenderle, pero creo que hubo maestros que batallaron mucho porque por su edad no están tan familiarizados con las tecnologías (Sujeto 1, CU).

Considero que esta pandemia nos dejó cosas buenas como aprender a usar las tecnologías para estudiar y para tener más y mejores opciones de información. Ahora creo que mis trabajos los hago más completos porqué ya puedo diferenciar documentos confiables, los analizo hasta comprender bien y eso también me ha ayudado a ser más crítico (Sujeto 26, CR).

En general, los estudiantes tienen las habilidades y competencias básicas para el uso de las TIC, como el manejo de la computadora, la búsqueda de información en la web y el uso de herramientas, sin embargo, se reconoce también el desarrollo de habilidades cognitivas como el análisis, pensamiento crítico y resolución de problemas que, en conjunto con las TIC, les han permitido enriquecer los conocimientos y mejorar sus tareas académicas.

\section{Retroalimentación por parte del docente.}

Esta subcategoría aborda las experiencias de los estudiantes en relación con la retroalimentación que recibían de sus profesores y qué tan pertinente fue para resolver sus dudas y lograr mejores aprendizajes. Al analizar las respuestas se detectan mayores carencias en los contextos rurales por 
la falta de condiciones de la mayoría de estudiantes y profesores para organizar clases sincrónicas a través de videollamadas, así como utilizar recursos y herramientas digitales como las plataformas, ocasionaron una falta de seguimiento a los aprendizajes pues la mayoría de los profesores solo mandaban correos o mensajes de texto indicando las actividades a realizar y, los que daban clases virtuales o videoconferencias lo hacían esporádicamente, se enfocaban en exponer y daban un tiempo muy reducido para aclarar dudas o ampliar contenidos, considerando además, que constantemente se interrumpía la clase por la falta de conectividad del internet.

En los contextos urbanos tuvieron clases sincrónicas y uso de plataformas educativas, pero los estudiantes manifiestan que no lograron los aprendizajes deseados, se quedaron con dudas o no recibieron una retroalimentación a profundidad de sus profesores.

Yo pienso que aprendí menos, porque entendía como podía y a mi manera y si tenía dudas me quedaba con ellas, no alcanzaba el tiempo para que el maestro nos respondiera a todos (Sujeto 4, CU).

En mi caso siento que no se me quedo nada, que no aprendí nada, me quede corta y con demasiadas dudas de todo. Mandaba todos los trabajos al correo de los profes pero no supe si estaban bien o no y los que me revisaban y me ponían comentarios, eran confusos y muchas veces no me quedaba claro en qué me había equivocado ni qué era lo correcto (Sujeto 24, CR).

La verdad aprendí menos, porque me quedé con dudas y no entendí algunas cosas de trabajos, solo los hice por obligación y ni siquiera supe para qué me serviría. Cuando estábamos en el salón era divertido y nos aclaraban las dudas en el momento, podíamos preguntar una y otra vez si no terminabamos de comprender, nuestros compañeros también aportaban y al final entre todos nos corregiamos o complementabamos nuestras ideas (Sujeto 22, CR).

Se puede identificar una retroalimentación insuficiente e ineficaz que provoca falta de motivación, interacción y aprendizaje de los estudiantes. Asimismo, se deja ver en la mayoría de las expresiones, que la retroalimentación es uno de los aspectos que más se valora en los ambientes virtuales.

\section{Habilidades del alumno para el autoaprendizaje.}

En esta subcategoría se analizan las percepciones de los participantes en relación con su capacidad para tomar el papel de protagonista en la construcción de sus conocimientos a partir de la autogestión, la organización de tiempos y tareas, el aprendizaje autónomo y la voluntad y capacidad de profundización y reflexión de los contenidos de los cursos.

Entre los resultados que descatan, en ambos contextos, se encuentra un desarrollo de la autonomía por parte de los estudiantes para indagar a profundidad en los temas que no fueron comprendidos, mayor capacidad crítica para seleccionar la información, analizarla y presentar los productos solicitados y, conforme avanzó el periodo escolar, mejoraron en la organización del tiempo y planificación para elaborar sus tareas escolares.

Aunque prefiero las clases presenciales, reconozco que esta experiencia me ha hecho valorar más a mis maestros y a mis compañeros y también ha sido positiva en cuanto a que te permite organizar tus tiempos de estudio y ser autodidácta (Sujeto $8, C U$ ).

Me gusta trabajar así, virtual, porque no pierdo tiempo en trasladarme a la escuela en camión, pero al principio me costó hacer las actividades por mi misma, dejaba todo para el último día y claro que no alcanzaba a terminar. Pero ahorita ya tengo mi horario cada día para la escuela y si me sobra tiempo me pongo a leer lo que no me quedó claro, entonces esta padre trabajar así (Sujeto 7, CU). 
Ha sido muy dificil esta situación porque como vivo en una comunidad donde no hay internet, tengo que bajar al pueblo, rentar computadora e internet para estar mandando los trabajos y sale muy caro. Y luego muchas cosas no entiendo porque todo el material es digital y no puedo estar rentando computadora todos los días para alcanzar a revisar todos los documentos, que a mi parecer eran exagerados en muchas ocasiones (Sujeto 27, CR)

Lo que más he aprendido ahora que las clases no son presenciales, es a ser más autodidacta. Ahora depende más de mí aprender algo que de mis maestros (Sujeto19, CR)

Aprender con una modalidad de educación virtual es mucha responsabilidad para nosotros como estudiantes. Yo he aprendido más sobre las TIC y cómo usarlas para mis tareas, también he aprendido a ser más organizada y más constante en lo que me propongo, también soy más reflexiva en lo que pasa a mi alrededor (Sujeto 23, CR).

La capacidad de organización y autodisciplina han significado un gran desafio para los estudiantes, según las entrevistas aplicadas, más del $60 \%$ manifiesta que, al inicio, fue de las habilidades donde hubo mayor dificultad, pero también de las que más valoran ahora que las desarrollaron. Por otro lado, el cambio de estrategias de aprendizaje llegó a percibirse, por más de la mitad de los entrevistados, como ineficiente, ausente de beneficios y con un menor acompañamiento por parte de los docentes. Pero también fue valorada y reconocida la importancia de aprender a ser autodidactas y de superarse por sí mismos.

\section{Condiciones emocionales para afrontar una metodología virtual de educación en un contexto global de pandemia.}

En esta categoría se identificaron dos subcategorías que permitieron conocer y comprender el sentir de los estudiantes ante este fenómeno inédito y cómo lo afrontaron emocional y actitudinalmente. Las subcategorías son Motivación y Situación emocional:

\section{Motivación.}

Se describe el sentir de los entrevistados en relación con el interés que mantuvieron o no para tomar sus clases en modalidad virtual, realizar las actividades asignadas por los profesores y, sobretodo, por aprender aún y cuando surgieron diversos factores que causaron adversidades tanto en la enseñanza como en el aprendizaje (didácticas tradicionales, saturación de tareas, retroalimentación insuficiente, problemas de conectividad, entre otros arriba mencionados).

Al inicio, cuando nos dijeron que tomaríamos las clases virtuales, fue emocionante porque era algo nuevo y pensé que sería divertido. Pero después de unas semanas de ver que las clases eran aburridas, que sólo nos encargaban leer y hacer ensayos, resúmenes, contestar cuestionarios y que además me estaba quedando con muchas dudas, mis expectativas bajaron y empecé a cumplir sólo para no reprobar (Sujeto 4, CU).

No me siento motivada, a mi me gusta estar cara a cara con compañeros y maestros, discutir sobre un tema, escuchar lo que otros piensan, que se hagan debates. Tampoco digo que me vale la escuela, al contrario, no he dejado de ser responsable, pero si me preguntan si disfruto mis clases y mi carrera, la respuesta es no (Sujeto 21, CR).

El semestre pasado que empezó todo lo de la pandemia, tenía miedo de salir y contagiarme, y tener clases en casa era bastante bueno pues me sentía segura. Pero en agosto que tuvimos que seguir así, empezó a ser frustrante porque siento que no doy el cien, que no aprendo igual, que ahora paso más tiempo haciendo tareas que antes que iba a presencial, además de que extraño el ambiente de un salón de clases. Me siento triste y desmotivada (Sujeto $25, C R)$. 
No estoy motivado, nada. De hecho, me quise dar de baja a principios de semestre porque siento que sólo estoy perdiendo el tiempo (Sujeto 19, CR).

La totalidad de los entrevistados manifiestan no estar motivados en sus estudios en esta modalidad virtual. Consideran que los aprendizajes no son adquiridos a pesar de que el tiempo dedicado es mayor que en presencial. Hay un desinterés y una sensación de pérdida de tiempo.

Según las percepciones de los estudiantes hay ausencia de los espacios para socializar, las tareas son excesivas y en ocasiones, poco significativas o descontextualizadas. Ser conscientes de esto permite a los docentes reconocer esas áreas de oportunidad para mejorar su práctica docente y por ende, favorecer los aprendizajes de sus estudiantes.

\section{Situación emocional.}

La subcategoría Situación emocional, describe las emociones más recurrentes de los estudiantes bajo esta modalidad virtual en un escenario de pandemia y confinamiento. Se mencionó ya la importancia de la autogestión del conocimiento, pero es también importante la gestión de las emociones para rendir académicamente. Ante este escenario, las personas, en general, se han visto afectadas por diversas situaciones como el desempleo, la pérdida de seres queridos a causa del Covid-19, el aislamiento social, la incertidumbre, etc., generando ansiedad, estrés, depresión, entre otros.

A partir de la revisión de los comentarios de los participantes, se identificó en todos ellos la presencia de ansiedad, estrés y, en mayor o menor medida, depresión. Tanto en el contexto rural como en el urbano han tenido de manera recurrente sentimientos como frustración, tristeza, enojo, desesperanza, sin embargo, es importante precisar que las causas son diferentes. Mientras que en contextos urbanos hay mayor preocupación por el aislamiento y el riesgo de contagio, en los contextos rurales el principal temor es la falta de conectividad y la situación económica.

La pandemia y estar encerrado en casa han incrementado mi estrés y ansiedad, no sólo por la pérdida de clases presenciales, la situación en sí, el saber que me puedo contagiar yo o un ser querido, no tengo cabeza para concentrarme, aunque sé que no puedo hacer nada, pero son pensamientos negativos que no puedo evitar (Sujeto 3, CU).

Cuando empezó la pandemia y cambiamos a modalidad virtual, cerraron lugares y todo se puso muy intenso, me la vivía con miedo, sobre todo porque mis papás tenían que seguir yendo a trabajar. Y luego a eso le sumamos que las clases virtuales fueron una decepción, que nos saturaban de tareas, que no podíamos ver a nuestros amigos, fueron meses muy estresantes (Sujeto 7, CU).

Tuve muchos sentimientos encontrados. Cuando nos mandaron a tomar las clases en casa pensé que era bueno para evitar el contagio, pero luego todo se complicó porque era necesario el internet para poder hacer todas las tareas y mandarlas a tiempo y donde vivo es muy lento y se va a cada rato (Sujeto 20, CR).

Creo que las emociones que más tuve fueron las de tristeza y miedo. Ah y frustración. Primero miedo por el Covid 19, luego mi papá quedó desempleado. En las clases empece a batallar por el internet. A veces no alcanzaba a hacer las tareas y empecé a tener problemas con algunos maestros (Sujeto 27, CR).

La falta de bienestar emocional refleja, como se observa en algunos de los entrevistados, dificultad para concentrarse y enfocarse en las tareas escolares, lo que a su vez repercute en el aprendizaje. Además, la insatisfacción de las clases virtuales, la percepción de que no hay aprendizaje significativo y que hay una pérdida de tiempo, así como la falta de interacciones con 
sus maestros y compañeros, han ocasionado, en los entrevistados, inseguridad y descontrol de su propia vida, que se traduce en enojo, tristeza y desinterés.

\section{Condiciones materiales y de recursos.}

En esta tercera y última categoría, se hace una descripción del número de estudiantes que cuentan con los recursos materiales indispensables para llevar sus clases en modalidad virtual de la mejor manera posible. Estos son equipo de cómputo, conectividad a internet y un espacio óptimo para estudiar, con buena iluminación, ajeno a distractores como el ruido y con mobiliario como silla y mesa/escritorio que aminore el cansancio de pasar tantas horas frente a una computadora.

Se encontró que en contexto urbano el $75 \%$ de los entrevistados contaban con computadora o laptop y el $68 \%$ tenían internet en casa, a diferencia de los contextos rurales, donde sólo el $42 \%$ tenía computadora o laptop y el $25 \%$ servicio de internet.

En relación con un espacio o cuarto de estudio con las condiciones físicas necesarias y sin distractores, se detecta que más del $60 \%$ del total de entrevistados realizan sus tareas o sus clases virtuales en áreas comunes de su casa como la sala o el comedor y comentan que suele ser incómodo porque hay otros miembros de la familia que ocupan esos espacios con otras actividades mientras ellos toman sus clases. El $40 \%$ dice que usa su habitación como área de estudio, pero sólo el $15 \%$ de ellos tienen el mobiliario necesario, los otros utilizan la cama para sentarse o acomodar la laptop. En el contexto rural más del 50\% de los estudiantes acuden con familiares, amigos o centros donde rentan computadoras e internet, al menos una vez a la semana, también comentan que las lecturas las descargan y leen en el celular para terminar a tiempo sus tareas, lo que les resulta aún más cansado.

\section{Discusión}

Se presenta a continuación un análisis entre la teoría y su relación con los resultados de este estudio:

Los estudiantes perciben las prácticas docentes como aburridas, pasivas, poco flexibles, expositivas y saturadas de información y actividades. Afirman que sus aprendizajes están por debajo de lo esperado pues no se profundizaba en los contenidos. De acuerdo con López, Lugo y Toranzos (2014) y, a la inversa de lo que mencionan los entrevistados, el docente virtual debe innovar en las decisiones pedagógicas tales como el diseño del procedimiento de las actividades, la presentación de contenidos atractivos y digeribles, los medios interactivos que permitan una retroalimentación y una comunicación oportuna, la evaluación como indicador fiable para identificar si se logró o no el aprendizaje, los espacios y las metodologías que promuevan el trabajo colectivo y colaborativo, entre otros.

Por otro lado, se manifiestan aspectos positivos como el desarrollo de la autodisciplina, la organización y el autoaprendizaje. Al respeto, Rugeles, Mora y Metaute (2015), refieren que el estudiante en modalidad virtual debe desarrollar la capacidad de autogestión a través de la autodisciplina, autoaprendizaje y fortalecimiento del análisis crítico y reflexivo.

La autodisciplina entendida como la fuerza de voluntad para cumplir con las propias metas; el autoaprendizaje, continuando con estos autores, refiere a la capacidad del sujeto para aprender de forma autónoma, activa y participativa; y, respecto al fortalecimiento del análisis crítico y reflexivo, se refiere a "la habilidad para razonar, analizar y argumentar hechos o acciones que facilitan el desarrollo integral del estudiante y la generación del conocimiento" $(2015, \mathrm{p}$. 135). El estudiante virtual debe asumir estas dimensiones para comprender no solo lo que esta en los textos, sino lo que ocurre a su alrededor, analizándolo, discerniéndolo y aplicándolo en diversos aspectos de su vida. 
En cuanto a la comunicación, el estudio refleja que ésta fue unilateral, carente de diálogo y de tipo instructivo más que reflexivo. La educación en estos ambientes virtuales debe caracterizarse por propiciar la interacción entre personas y entre persona-contenido. Al respecto, "el rol del docente es desarrollar contenidos interactivos que faciliten la participación del estudiante, y que le permitan crear el conocimiento y desarrollar sus habilidades comunicativas" (Castro, 2016, p. 103).

En ideas similares, Fernández, Chamizo y Sánchez (2021) encuentran que de los inconvenientes más recurrentes a la hora de comunicarse profesores y estudiantes en una modalidad virtual y/o a distancia son "la falta de estímulo del alumnado al no encontrarse en el aula, la consecuente dificultad a la hora de implicar al estudiante y de conectar emocionalmente con él, así como los problemas derivados de la conexión a la red" (p. 165).

Sin embargo, se rescata en los comentarios que las clases sincrónicas mediante las videollamadas fueron valoradas positivamente ya que favorecieron la comunicación y permitieron mayor acercamiento con maestros y compañeros. Tal como menciona Viloria y Hamburger (2019), las video conferencias fomentan los vínculos entre profesor-alumno al permitir una comunicación más cercana y directa, promueven una retroalimentación al momento de la duda y es de las herramientas que mejor han funcionado en la enseñanza y el aprendizaje.

Respecto al manejo de las TIC, se observa un buen desempeño, sobre todo de los estudiantes, quienes afirman tener las competencias y habilidades para utilizarlas o, en su defecto, aprenderlas sin mayor problema. Los profesores, aún y cuando algunos tuvieron dificultades, también han ido mejorando. Lo que se resalta en este aspecto, es que los entrevistados refieren que usar las TIC les ha favorecido en el desarrollo de otras habilidades de tipo cognitivo, como resolver dudas por su cuenta, analizar textos, identificar información confiable, ser más críticos y reflexivos de aquello que ocurre a su alrededor. Erstad y Voogt (2018) citados en Almerich, Suárez, Díaz y Natividad (2020), afirman que las competencias en TIC y las de alta habilidad cognitiva (resolución de problemas, pensamiento crítico, adaptabilidad, aprendizaje autogestionado y otras) no se pueden enseñar y aprender por separado, hay una correlación positiva entre ambas y la tecnología es un facilitador del pensamiento superior.

Sobre la retroalimentación, hubo coincidencia entre los entrevistados que fue deficiente, no permitía aclarar dudas o profundizar en algún contenido e incluso, en ocasiones, estuvo ausente. Bonnel (2008), señala que ésta no consiste en usar frases como "buen trabajo, bien hecho, interesantes reflexiones, etc." pues no hay profundización que permitan mejorar o generen la autoreflexión y los procesos metacognitivos en los estudiantes. Kopp, Germ y Mandl (2010) afirman que el rol del docente en la educación virtual es informar a sus estudiantes oportunamente sobre los logros en su nivel de desempeño, los objetivos de aprendizaje alcanzados o no y las recomendaciones de estrategias de aprendizaje que les ayuden a profundizar y reconocer sus avances y sus necesidades.

La motivación, ausente en todos los participantes, es un elemento clave para el aprendizaje. Los entrevistados manifiestan que la falta de innovación y contextualización de las clases, la saturación de información y actividades poco relevantes, la ausencia de reflexión y profundización en los contenidos, entre otras, les han llevado a no tener interés ni motivación. Sobre ello, Selvi (2010) afirma que la motivación esta relacionada con el proceso de enseñanzaaprendizaje, la participación y atención, el entorno de aprendizaje en línea y la gestión de tiempo. En ideas similares, Kim y Frick (2011), refieren la sobrecarga cognitiva, la dificultad percibida de las tareas a realizar, la falta de recursos propios para regular el autoaprendizaje, la ausencia de interacciones sociales, entre otros, como causantes del desinterés e insatisfacción de los sujetos. 
Al respecto, Hartnett (2018) menciona que la falta de contacto presencial hace más difícil a los profesores detectar si los estudiantes están aburridos o desinteresados y, por otro lado, el anonimato ocasiona en los estudiantes una sensación de libertad para relajarse cuando lo deseen.

La importancia de promover la motivación es determinante para evitar la deserción y lograr los aprendizajes esperados. Sobre esto, Selvi (2010) propone factores como el entusiasmo de profesores, la selección de materiales y contenidos relevantes y significativos, los espacios en común que permitan la participación activa de los estudiantes, dificultad en las tareas que se perciba como reto y no como un imposible, instrucciones precisas y claras, etc. Si el estudiante no esta motivado, no aprende, tal como dice Maturana (2001), lo humano se constituye en la unión de lo emocional con lo racional, es decir, todas nuestras acciones tienen un fundamento emocional.

En ideas similares, se encuentra la situación emocional de los estudiantes. Sentimientos como la desesperanza, la tristeza, el miedo y la frustración, estan presentes de manera recurrente en ellos. Al respecto, Brooks et al. (2020), mencionan que esta situación de pandemia genera en los estudiantes una sensación de sentirse atrapado, miedo al virus, aburrimiento, alimentación inadecuada, sedentarismo, entre otras, que se refleja en su aspecto emocional, físico y cognitivo.

Respecto a los recursos tecnológicos y materiales, son un problema principalmente para los estudiantes en contextos rurales. La conectividad en estas comunidades es deficiente y por ende, la calidad de los aprendizajes, de la motivación y del interés de los estudiantes se ve afectada. En el informe Desarrollo Humano y Covid-19 en México: desafios para una recuperación sostenible, muestra que solo el $16 \%$ de familias de estrato bajo cuenta con computadora y conexión a internet, mientras que el estrato alto, es de 79\%. Esto permite entender el problema que enfrentan los estudiantes de escasos recursos, de comunidades rurales e indígenas para acceder a una educación virtual, donde por consecuencia, se afecta su aprendizaje e incluso ocasiona la deserción escolar (Gallegos, 2021).

\section{Conclusiones}

La educación en tiempos de pandemia ha resultado un desafio para todo el sistema escolar. Entrar en una modalidad virtual debido a una urgencia sanitaria hizo evidente la ausencia de fundamentos teóricos y metodológicos en las prácticas docentes, que plantea esta modalidad. Los profesores utilizan las herramientas que tienen a su alcance y que mejor dominan para reproducir su práctica como lo harían en lo presencial, según el reporte del Observatorio de Educación Superior y Políticas Universitarias (OESPU, 2020).

Es un compromiso y una responsabilidad de las universidades ofrecer ambientes educativos virtuales que atiendan a la diversidad de estudiantes y que garanticen la accesibilidad y la equidad (Coleman y Berge, 2018). Asimismo, ofrecer una formación docente que le brinde competencias para adaptar y ajustar las estrategias metodológicas no sólo en lo pedagógico, sino que también considere el incremento de la motivación y la participación de los estudiantes y que atiendan las necesidades de la sociedad actual (Hurtado, 2020).

Con base en lo resultados obtenidos y en la revisión de literatura sobre el tema, los entornos virtuales aún y cuando posibilitan mayor acceso a la educación y a la información, y favorecen la autonomía de los estudiantes, continua siendo un reto para las universidades, pues las experiencias hasta ahora vividas por docentes y dicentes, permite dar cuenta de vacíos que obstaculizan el proceso de enseñanza-aprendizaje, y que no sólo tienen que ver con los profesores, sino con el mismo contexto de los estudiantes, las políticas educativas, las condiciones geográficas y económicas de las familias, entre otros. 
Para finalizar, la pandemia obligó a todas las escuelas, sobre todo de nivel medio y superior, a cambiar las clases presenciales por virtuales, pero también es cierto que saber utilizar las nuevas tecnologías y adquirir conocimientos, habilidades y actitudes para enseñar y aprender en ambientes virtuales, es una necesidad que demanda la sociedad actual y que compete a todos, maestros, alumnos y autoridades, adaptarnos al cambio y dar respuesta a las características de la realidad que nos toca vivir.

\section{Referencias}

Almerich, G., Suárez, J., Díaz, I. \& Natividad, O. (2020). Estructura de las competencias del siglo XXI en alumnado del ámbito educativo. Factores personales influyentes. Educación XXI, 23(1), 45-74. https://doi.org/10.5944/educXX1.23853

Aparicio, O. \& Ostos, O. (2021). Pedagogías emergentes en ambientes virtuales de aprendizaje. Revista internacional de pedagogía e innovación educativa. 1(1), 11-36. https://editic.net/ripie/index.php/ripie/article/view/25/20

Arango, Y., Díaz, P., y Zuleta, A. (2018). La inclusión digital en los sistemas de educación superior en la modalidad virtual. En Orozco, E., Rodríguez, J., Gómez, C., \& Barrietos, A. (Eds.), Cultura digital y las nuevas políticas educativas (pp. 15-26). Gedisa.

Bonnel, W. (2008). Improving feedback to students in online courses. Nursing Education Perspectives, 29(5), 290-294. https://pdfs.semanticscholar.org/98e4/08e10f0f21b7ee60af1f275113f48e1f9fb2.pdf

Brooks, S., Webster, R., Smith, L., Woodland, L., Wessely, S., Greenberg, N. \& Rubin, G. (2020). The psychological impact of quarantine and how to reduce it: rapid review of the evidence. Lancet, 395, 912 - 920. https://doi.org/10.1016/ S0140-6736(20)30460-8

Castro, M. (2016). La comunicación en la educación virtual y los desafíos de la relación docente

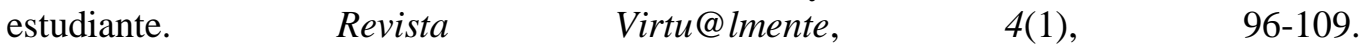
https://doi.org/10.21158/2357514x.v4.n1.2016.1524

CEPAL-UNESCO (2020). La educación en tiempos de la pandemia de COVID-19. Informe COVID-19 CEPAL-UNESCO. https://repositorio.cepal.org/bitstream/handle/11362/45904/1/S2000510_es.pdf

Coleman, M. \& Berge, Z. (2018). A Review of Accessibility in Online Higher Education. Online Journal of Distance Learning Administration, 21(1). https://www.westga.edu/ distance/ojdla/spring211/coleman_berge211.html

Díaz, P., Andrade, Y., Hincapié, A. y Uribe, A. (2021). Análisis del proceso metodológico en programas de educación superior en modalidad virtual. Revista de educación a distancia, 21(65). https://doi.org/10.6018/red.450711

Fernández, M., Chamizo, R. y Sánchez, R. (2021). Universidad y pandemia: comunicación en la educación a distancia. Ámbitos. Revista Internacional de Comunicación, 52, 156-174. https://dx.doi.org/10.12795/Ambitos.2021.i52.10 I

Gallegos, O. (2021). La educación virtual sin conectividad aumenta la desigualdad educativa. Cuadernos froterizos, (Núm. $\quad$ Especial), 21-27. http://erevistas.uacj.mx/ojs/index.php/cuadfront/article/view/4028/3366

Guzmán, R., Vázquez, J. y Escamilla, A. (2020). Cambio de paradigma en la educación. Cirujano General, 42(2), 132-137. https://dx.doi.org/10.35366/95373

Hartnett, M. (2018). Motivation in Distance Education. En Moore, M. y Diehl, W. (Ed.) Handbook of Distance Education. Routledge.

Hurtado, F. (2020). La educación en tiempos de pandemia: los desafios de la escuela del siglo XXI. CIEG, Revista arbitrada del Centro de Investigación y Estudios Gerenciales, (44), 174-187.

https://www.grupocieg.org/archivos_revista/Ed.44(176187)\%20Hurtado\%20Tavalera_a rticulo_id650.pdf

Kim, K. y Frick, T. (2011). Cambios en la motivación de los estudiantes durante el aprendizaje en línea. Revista de investigación en informática educativa, 44(1), 1-23. https://doi.org/10.2190/EC.44.1.a 
Kopp, B., Germ, M., y Mandl, H. (2010). Supporting virtual learning through e-tutoring. In Ecollaborative knowledge construction. En Ertl (Ed), Learning from computer-supported and virtual environments (pp. 213-231). Information Science Reference, Hershey, PA. Llafuente

Lister, M. (2014). Trends in the design of E-learning and online learning. Journal of Online Learning and Teaching, 10(4), 671-680. https://jolt.merlot.org/vol10no4/Lister_1214.pdf

López, N.; Lugo, M. y Toranzos, L. (2014). Políticas TIC en los sistemas educativos de América Latina. Informe sobre tendencias sociales y educativas en América Latina, 2014. Buenos Aires: IIPE-UNESCO-OEI

Maturana, H. (2001). Emociones y lenguaje en educación y política. Santiago de Chile: Ediciones Dolmen.

Observatorio de Educación Superior y Políticas Universitarias (2020, julio). La pandemia COVID19 y la Universidad: ¿Quo vadis? Boletín Informativo UNSAM. http://www.unsam.edu.ar/news/interna/OESPU-Boletin-informativo-2.pdf

Pérez, C., Suárez, R. y Rosillo, N. (2018). La educación virtual interactiva, el paradigma del futuro. Atenas, 4(44), https://www.redalyc.org/journal/4780/478055154009/html/

Ruiz, G. (2020). Covid-19: pensar la educación en un escenario inédito. Revista mexicana de $\begin{array}{lll}\text { investigación educativa, 229(85), } & \text { 2237. }\end{array}$ http://www.scielo.org.mx/pdf/rmie/v25n85/1405-6666-rmie-25-85-229.pdf

Reimers, F. y Schleicher, A. (2020). Un marco para guiar una respuesta educativa a la pandemia 2020 del Covid-19. Enseña Perú. https://globaled.gse.harvard.edu/files/geii/files/un_marco_para_guiar_una_respuesta_ed ucativa_a_la_pandemia_del_2020_del_covid-19_.pdf

Rugeles, P., Mora, B. y Metaute, P. (2015). El rol del estudiante en los ambientes educativos mediados por las TIC. Revista lasallista de investigación, 12(2), 132-138. http://www.scielo.org.co/pdf/rlsi/v12n2/v12n2a14.pdf

Selvi, K. (2010). Teachers' Competencies. Cultura International Journal of Philosophy of Culture and Axiology, 7(1), 167-175. http://doi.dx.org/10.5840/cultura20107133.

Viloria, H. y Hamburger, J. (2019). Uso de las herramientas comunicativas en los entornos virtuales de aprendizaje. Chasqui. Revista Latinoamericana de Comunicación, 140, $367-$ 384. https://doi.org/10.16921/chasqui.v0i140.3558 